\title{
Main care to avoid complications associated with thyroidectomy
}

\begin{abstract}
General objective: To identify the main care in patients undergoing total or partial thyroidectomy to avoid complications associated with surgery.

Methodology: Articles were searched in the databases: Pubmed, CINAHL y Cochrane Library Plus, with the free terms: laryngotracheal stenosis, tracheal stenosis, complications, surgery and care. Limited in Indian: English or Spanish, +18 years and published in the last 10 years.

Results: Out of a total of 140 articles found, 30 met the inclusion criteria of the finally selected topics. 17 that dealt with the stated objective and that were added to a 2002 article because of its relevance. Hyperthyroidism affects $1-3 \%$ of the general population and is usually more common in women. There are different treatment options available depending on their more or less invasive individualization. Surgery offers an early remission of endocrine disorder and more stable than more conservative strategies and also an earlier detection of thyroid cancer. Hypocalcemia is the most frequent, symptomatic or asymptomatic, rebleeding with hematoma, laryngeal nerve injury, acute pain or infection as the most important. There is a series of care that favors the early detection of these and other things.

Conclusion: This review has been made with the aim of knowing more about the most frequent complications after a thyroidectomy and establishing the basic learning needs that patients should know at discharge to take responsibility for their health. In addition, a series of recommendations has been made to patients at discharge to empower the patient to be responsible for their health and to recognize early complications, know and take responsibility for medication, know how to heal the wound, etc.
\end{abstract}

Keywords: thyroidectomy surgery, complications, postoperative and intervention
Volume 3 Issue 5 - 2019

\section{Cristina Subirana Ferrés, Isabel Diaz Martos, Nuria Romero Navarrete, Piedad Ruiz Barrio, Carlos Déniz Armangol, Fran Rivas Doyague \\ Medical area and thoracic surgery, Hospital Universitario de} Bellvitge, Spain

Correspondence: Cristina Subirana Ferrés, Medical area and thoracic surgery, Hospital Universitario de Bellvitge, Spain, Tel 932607575 EXT 2568, Email csubirana@bellvitgehospital.cat

Received: October 04, 2019 | Published: October 29, 2019
Abbreviations: VHI, vocal disability index; PTH, parathyroid hormone;

\section{Introduction}

Hyperthyroidism is understood as the excessive secretion of thyroid hormones, both from T3 (triiodothyronine) and T4 (tetraiodothyronine). The main symptoms of hyperthyroidism are associated with: asthenia, weight loss, anxiety, palpitations, temperature changes and changes in sleep pattern. They may or may not be accompanied by the presence of goiter and exophthalmos, which is suggestive of this metabolic disorder. Hyperthyroidism affects between $1-3 \%$ of the general population and is usually more frequent than affects women. The most frequent is Graves' disease, which represents $50-80 \%$ of cases of hyperthyroidism. ${ }^{1}$ There are different treatment options available depending on your more or less invasive individualization: anti-thyroid drugs, radioactive iodine, ethanol injection or surgery. According to the Ministry of Health and Consumption and Social Welfare, the procedures on thyroid, parathyroid and thyroglossal tract have a low percentage of mortality of $0.03 \%$ compared to other surgical procedures. ${ }^{2}$ Surgery offers an early remission of endocrine disorder and more stable than more conservative strategies and also an earlier detection of thyroid cancer. ${ }^{1}$ Goiter recurrence remains a problem that sometimes cannot be resolved with thyroxine suppressive therapy and primary surgery is a more aggressive but more effective way to prevent relapse. When a complete thyroidectomy is necessary, in the case of a recurrent toxic goiter, for example, re-interventions should be performed in highlevel surgical centers. ${ }^{3}$

As all surgery is not risk-free, there are a series of known and frequent postoperative complications such as hypocalcaemia (this being the most frequent), the presence of resuscitation producing a hematoma with less or greater severity or recurrent laryngeal nerve paralysis, especially after a total thyroidectomy. ${ }^{1-7}$ The relationship between the patient and the rate of postoperative complications is complex and is influenced by the intrinsic factors of the disease, the comorbidities of the patient and the same surgical treatment. ${ }^{5,6}$ There are several factors that can influence, such as age, race, functional status, smoking history, etc. In addition, the presence of thyroid malignancy was identified as indicative of increased postoperative complications. ${ }^{4}$ Currently, there are several strategies to address the treatment of hypocalcemia. Several studies have demonstrated the efficacy of prophylactic calcium and vitamin D, as well as the assessment of parathyroid hormone. ${ }^{8}$ Elderly patients presented a similar rate of complications compared to young patients, including 
transient hypocalcemia and paresis of the vocal cords, but nevertheless presented a higher rate of hospital readmission. (4.5\% vs. $1.2 \%){ }^{9}$

Swallowing disorders also occur after a thyroidectomy may be caused by the lesion of the superior laryngeal or inferior nerve, there are studies ${ }^{10}$ that show incidence of permanent unilateral lesions of the laryngeal nerve, manifesting with persistent hoarseness. ${ }^{11}$ The laryngeal nerves are responsible for the motility of the cricothyroid muscle, which favors the tension between the vocal cords and their injury is also related to swallowing symptoms. This symptomatology can be quite annoying until long after the intervention. ${ }^{12}$ A simple test that can be performed for diagnosis is the vision of the vocal cords through the laryngoscope and will show us any abnormal mobility of these. They can also appear not only swallowing disorders but also voice. Vicente et al. ${ }^{13}$ determines factors that can be evaluated practically in the early postoperative period and at-risk patients can be educated about their functional recovery and be referred to voice and language specialists to prevent the development of mechanisms of maladaptive compensation, thus improving the general attention of the patient. In general, we should know that adequate and standardized nursing care in those postoperative patients of a thyroidectomy will help us reduce the complications and anxiety of the patients as well as provide security in our care. The reason for this review was to reduce inappropriate variations in clinical practice, promote quality care, based on the most up-to-date evidence so far. For this reason, the objective of this review is to identify those main cares in patients undergoing a thyroidectomy to reduce the complications associated with this surgical intervention.

\section{Methodology}

In order to evaluate the studies and determine the nursing care of patients who underwent a partial or total sea thyroidectomy and reduce the associated complications, a literature review of the literature published from 2008 to the present in English and Spanish was made. This narrative review was reviewed between July 2018 and finalized in September 2018. The databases accessed were Pubmed, CINHAL and Cochrane Library. The search was conducted using free language, using these keywords: thyroidectomy surgery, complications, postoperative and intervention. In the field (title, summary and / or abstract). The use of a natural language was considered necessary because it is a subject with little evidence regarding nursing care. The search is complemented by specific queries to certain internet web pages: Ministry of Health, Consumer Affairs and Social Welfare (Spain). The truncations used in the search, carried out in July 2018 until September, are the following: Terms used in the search (Figure $1)$.

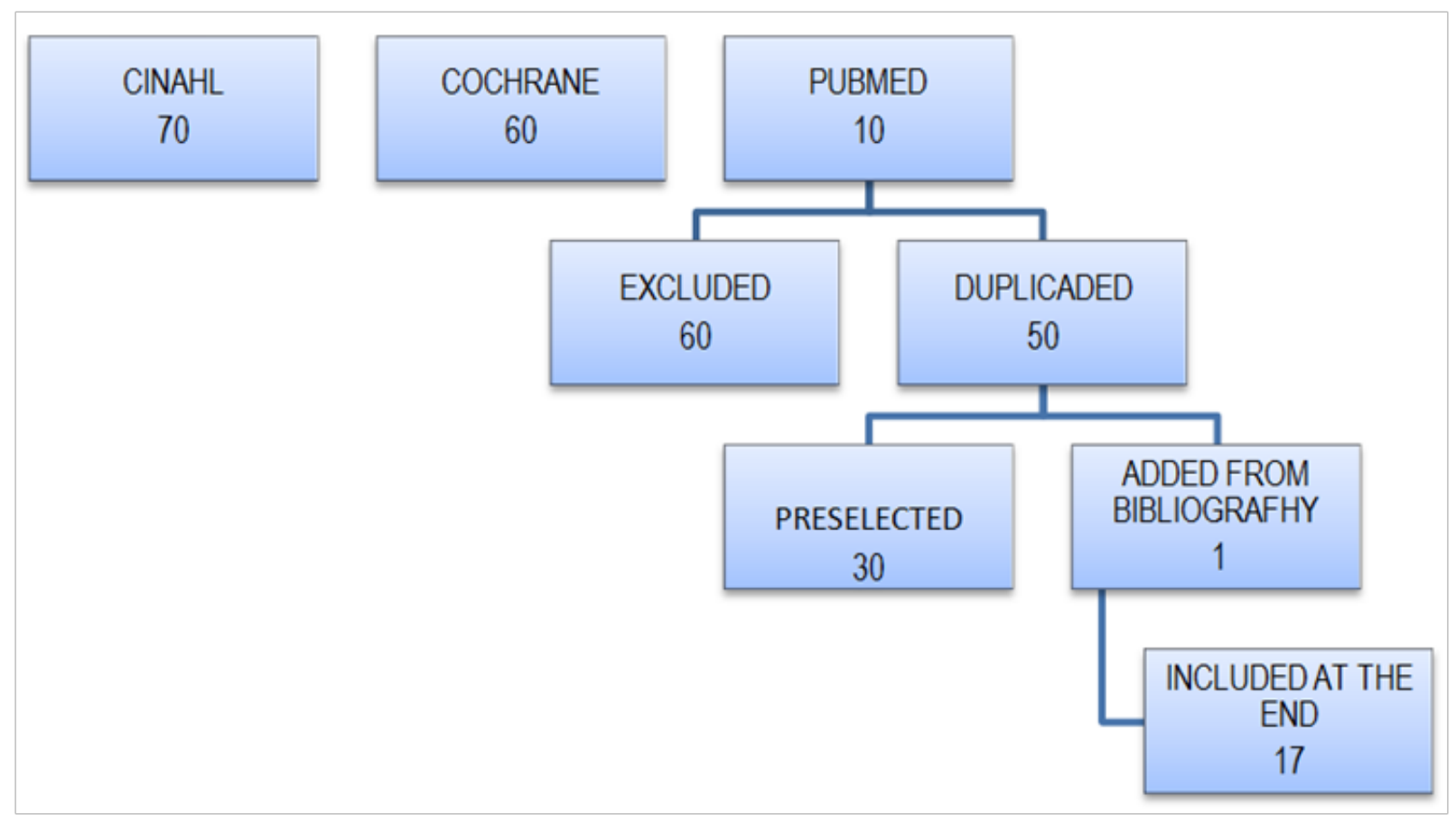

Figure I Diagram of the literature review process and article selection.

$\begin{array}{ll}\text { PUBMED } & \begin{array}{l}\text { "thyroidectomy surgery" AND complications "thyroidectomy surgery" AND postoperative "thyroidectomy } \\ \text { surgery" AND interventions }\end{array} \\ \text { CINAHL } & \begin{array}{l}\text { thyroidectomy surgery AND complications thyroidectomy surgery AND postoperative thyroidectomy surgery } \\ \text { AND interventions }\end{array}\end{array}$

COCHRANE thyroidectomy surgery AND complications AND intervention 
he filters were applied to the articles: last 10 years (2008-2018), articles only in Spanish or English, human species, ages over 18 years, as well as being able to access the full texts and not only the Abstract. For those items that could not be obtained directly, they were searched through the Barcelona University platform.

\section{Extraction of the data obtained}

After the search, all documents in which at least the summary could

Table I Criteria for inclusion and exclusion of search in databases be accessed were read and evaluated (preselected), subsequently, a list was made with these and only those that met the criteria previously cited to be included were finally selected (Table 1) and of sufficient relevance for the review. The Critical Appraisal Skills Program (CASPe) guide ${ }^{14}$ was used to critically assess the documents obtained. Also included was an article that referred to other articles in its bibliography because of its relevance.

Inclusion criteria
- Population over I 8 years old
- Post operative complications
- Articles that talk about the morbidity influencing factors after
thyroid surgery.
- Articles that will analyze the hormonal level after the
postoperative period.
- Articles that will talk about swallowing and voice problems that
may occur after thyroidectomy intervention.
- Complications that may appear after hospitalization o during it

\section{Basic risk assessment}

The methodological quality of the included studies was independently assessed. The levels of evidence of the Joanna Briggs Institute Levels of Evidence ${ }^{15}$ were used to assess the degree/level of evidence, from level 1 to level 5 according to the type of study design of each selected article. An analysis of the most mentioned complications was carried out in all the selected studies and the care related to these complications was developed, grouping the care within the complication that was most related to it.

\section{Exclusion criteria}

-No articles comparing surgical techniques (invasive vs. non-invasive) for the approach of the thyroid incision.

- No articles dealing with complications or intraoperative or preoperative interventions.

- No combinations of surgeries (cardiac and thyroidectomy, for example).

Table 2 Inclusion criteria of 10 Pubmed, 70 CINAHL, 60 Cochrane

\begin{tabular}{|c|c|c|c|}
\hline Authors and year & $\begin{array}{l}\text { Methodology of } \\
\text { the study }\end{array}$ & Subjects & Results \\
\hline \multirow[t]{5}{*}{ C Quéralt et al.' } & Retrospective study & $N=200$ & - There were no deaths during follow-up \\
\hline & & & $\begin{array}{l}\text { - I } 8 \text { patients with postoperative hematoma, } 17 \% \text { were treated with anti } \\
\text { coagulation compared to } 13 \% \text { of patients without. }\end{array}$ \\
\hline & & & $\begin{array}{l}\text { - Recurrent laryngeal nerve paralysis, } 4 \text { patients already presented it previously, } \\
\text { I } 3 \text { patients presented it after the operation, } 5 \text { recovered normal laryngeal } \\
\text { movement within the year. }\end{array}$ \\
\hline & & & $\begin{array}{l}\text { - } 20 \% \text { of patients had hypokalemia due to } \mathrm{D} 2 \text {, definitive only } 3 \% .9 \% \text { of the } \\
\text { patients with parathyroid resection developed definite hypocellcaemia. }\end{array}$ \\
\hline & & & - After surgery only 2 cases of recurrence \\
\hline Miccoli G et al. ${ }^{3}$ & $\begin{array}{l}\text { Prospective } \\
\text { observational study }\end{array}$ & $N=214$ & $\begin{array}{l}\text { - After the re-intervention there was temporary hypoparathyroidism in } 37.7 \% \text { of } \\
\text { the patients, and definitive hypoparathyroidism occurred in } 7.2 \% \text {. }\end{array}$ \\
\hline & & & $\begin{array}{l}\text { - The recurrent transient paralysis of the laryngeal nerve occurred in } 3.3 \% \text { of } \\
\text { patients and permanent nerve paralysis in } 0.4 \% \text {. In } 1.9 \% \text { of patients, the revision } \\
\text { of hemostasis was necessary for postoperative hemorrhage. }\end{array}$ \\
\hline Cauley et al. ${ }^{4}$ & $\begin{array}{l}\text { Retrospective cohort } \\
\text { study }\end{array}$ & $N=40.025$ & $\begin{array}{l}\text { - Complications at } 30 \text { days, of the total number of patients undergoing a } \\
\text { total thyroidectomy was } 7.74 \% \text {. }\end{array}$ \\
\hline
\end{tabular}


Table continued

\begin{tabular}{|c|c|c|c|}
\hline Authors and year & $\begin{array}{l}\text { Methodology of } \\
\text { the study }\end{array}$ & Subjects & Results \\
\hline \multirow{5}{*}{ Balentine et al. ${ }^{5}$} & \multirow{5}{*}{$\begin{array}{l}\text { Sistematic review of } \\
35 \text { studies }\end{array}$} & \multirow{5}{*}{$\begin{array}{l}\text { Outpatient } \\
\text { thyroidectomy; } \\
\text { Same day } \\
\text { thyroidectomy; } \\
\text { Short stay } \\
\text { thyroidectomy; } \\
\text { Safety; } \\
\text { Hematoma }\end{array}$} & $\begin{array}{l}\text { Preoperative factors that affected complication rates for thyroidectomies } \\
\text { in hospitalized patients included: age } \geq 70 \text { years, non-Caucasian, functional } \\
\text { dependent status, history of congestive heart failure, history of smoking, bleeding } \\
\text { disorder, wound infection and preoperative sepsis. }\end{array}$ \\
\hline & & & $\begin{array}{l}\text { - Thyroid malignant pathology is associated with increased vascularization, } \\
\text { immunosuppression and poor wound healing. Presence of malignancy was } \\
\text { identified as a predictor of increased postoperative complications }\end{array}$ \\
\hline & & & $\begin{array}{l}\text { Risks of a thyroidectomy: Hematoma, Hypoparathyroidism, Recurrent } \\
\text { lesion of the laryngeal nerve. }\end{array}$ \\
\hline & & & $\begin{array}{l}\text { Symptomatology and complications to which you must be alert to notify } \\
\text { the surgeon. }\end{array}$ \\
\hline & & & - $\quad$ Techniques to improve the success of thyroid surgery \\
\hline \multirow[t]{3}{*}{ Bergenfelz et al. ${ }^{6}$} & \multirow[t]{3}{*}{$\begin{array}{l}\text { Prospective } \\
\text { controlled clinical } \\
\text { trial }\end{array}$} & $N=3.660$ & $\begin{array}{l}\text { - } \quad \text { After thyroidectomy, a new hemorrhage occurred in } 2.1 \% \text { and was } \\
\text { associated with a more advanced age and male sex. }\end{array}$ \\
\hline & & & $\begin{array}{l}\text { Postoperative wound infection occurred in } 1.6 \% \text { and is associated with } \\
\text { lymph node dissection vs. those that were not performed. }\end{array}$ \\
\hline & & & $\begin{array}{l}\text { Unilateral paralysis of the recurrent laryngeal nerve was diagnosed in } \\
3.9 \% \text { and bilateral in } 0.2 \% \text {. It was associated with advanced age, intrathoracic } \\
\text { goiter, thyrotoxicosis. }\end{array}$ \\
\hline
\end{tabular}

- After a total thyroidectomy, hypocalcaemia occurred in $9.9 \%$ that had to be treated with vitamin $\mathrm{D}$ and fell to $4.4 \%$ in the 6 -month follow-up

Trottier et al. ${ }^{7}$

Carter et al. ${ }^{8}$

Efremidou et al. ${ }^{10}$

Prospective non-

Melanie et al. ${ }^{9}$

\section{Descriptive}

retrospective study
Retrospective study

$N=932$

$N=232$

Retrospective study Prospective Protocol

$N=620$ study

\section{- Complications: $2.5 \%$ Hypocalcaemia, $0.4 \%$ Hematoma, $0.4 \%$ vocal cord} injury, $0.9 \%$ wound infection and $0.4 \%$ acute pain.

- $\quad$ The main symptom for the detection of hypocalcemia was tingling. The symptoms were not permanent and no patient had severe symptoms such as seizures or tetany.

- Vocal cord injury can be experienced by persistent hoarseness

- $\quad$ Using the protocol, $6 \mathrm{I}$ patients (I0.2\%) developed hypocalcaemia but never showed symptoms and 24 (3.9\%) developed advanced hypocalcaemia. Both groups were similar in terms of sex, diagnosis of cancer and preoperative calcium and PTH.The group with advanced hypocalcaemia was younger, with lower postoperative PTH levels

$33 \%$ of patients with advanced hypocalcaemia had a PTH $\leq 5 \mathrm{pg} / \mathrm{ml}$ compared to only $6 \%$ asymptomatic patients. These patients required a dose increase of calcitriol to achieve biochemical correction and symptom relief

- $\quad$ The incidence of permanent bilateral recurrent paralysis of the laryngeal nerve was $0 \%$ and the permanent unilateral recurrent laryngeal nerve paralysis was $0.2 \%$, while the incidence of unilateral recurrent paralysis of the laryngeal nerve was $1.3 \%$.

Permanent hypocalcemia occurred in $0.3 \%$ and general temporal hypocalcemia occurred in $7.3 \%$ of patients. Hemorrhage that requires repeated surgery occurred in $0.2 \%$ of patients.

There was no wound infection, and postoperative mortality was $0 \%$. We do not observe disease recurrence during a median follow-up of 9 (range 2-20) years.

- $\quad$ Elderly patients presented a similar rate of complications compared to young patients, including transient hypocalcemia (I $2.5 \%$ vs. I I. I\%, respectively) and temporary vocal cord parresia ( $2.9 \%$ vs. $3.9 \%)$, but a higher readmission rate $(4.5 \%$ vs. I. $2 \%)$. 
Table continued

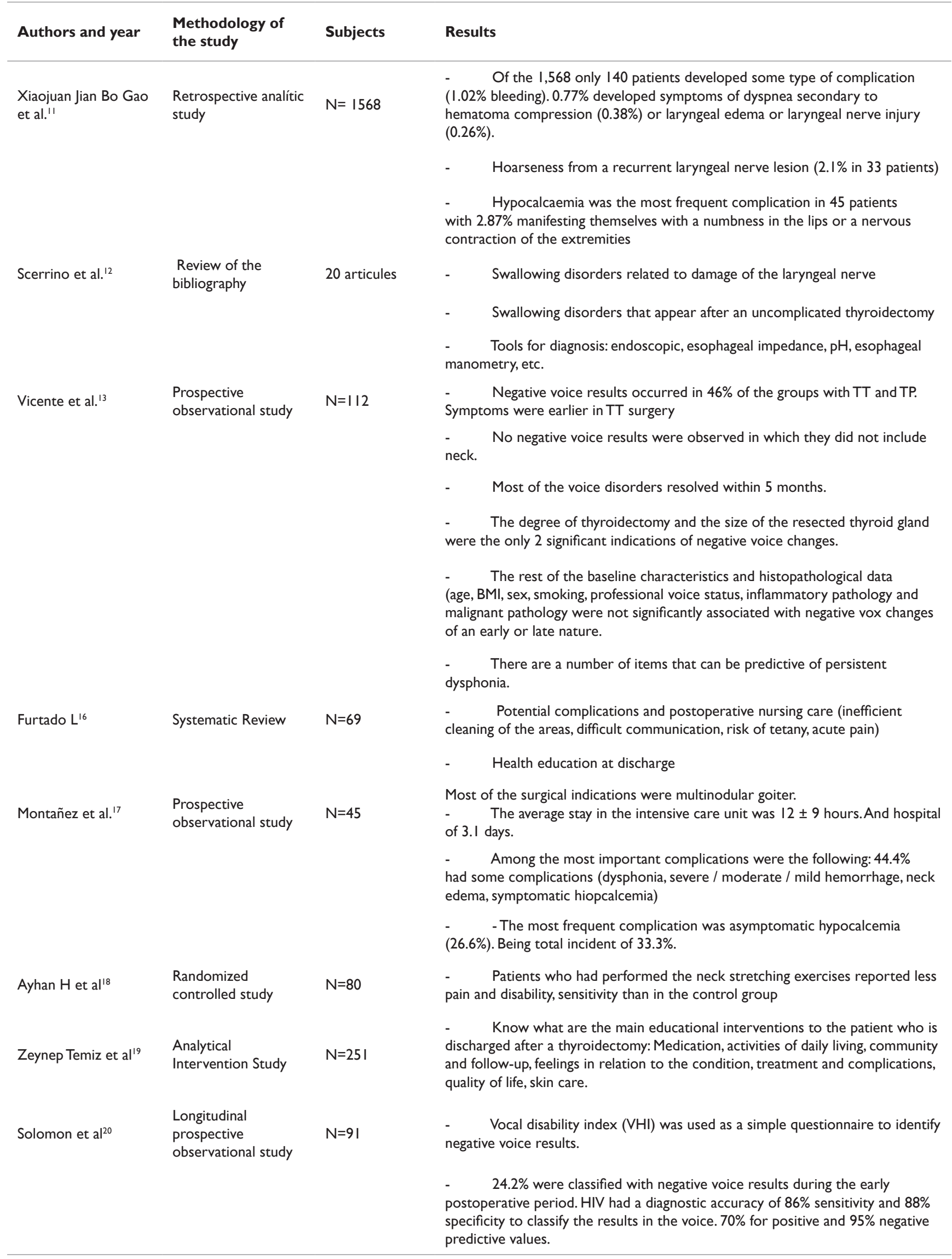




\section{Prevention of complications and nursing care in post- surgical management}

As mentioned above, thyroidectomy is considered a safe surgery because it as a low percentage of complications, but it is worth highlighting which are to know their care and prevent them.

\section{Risk of bleeding and bruising $(5$, I I, I 6)}

I. Control of the wound and the area due to the risk of hematoma. Both the front and back of the neck. Assess the degree of severity of the hematoma. Assess if it is a serious situation and handle your observation. The change in the circumference of the neck ${ }^{16}$ may be an indication of hematoma. According to Balentine and Sippel $^{5}$ some bruises develop after an observation period of 6 hours or 23. It is not clear what the optimal time for observation is. Some studies show bruises that develop more than 1 week after surgery; therefore, the patient must be educated because it may not occur during the hospitalization period. Above all, special attention should be given to those patients taking anticoagulant treatment. In addition, performing a lymph node dissection may also increase the risk of bleeding.

II. Assess analytical levels of coagulation parameters.

III. Adapt the diet and observe its tolerance.

In the postoperative period, the diet should be cold and not too hot in order to avoid overheating that it could induce in the blood vessels of the neck and dilate more easily and increase the risk of bleeding. Start tolerance with warm water and progress if there are no signs of dysphagia. ${ }^{11}$ Xiaojuan Jian et al. ${ }^{11}$ in his study defined a series of symptoms for the early detection of bleeding. Observe the presence of neck thickening, subcutaneous congestion, hemorrhagic exudation in wound dressings, sudden increase in drainage fluid in bright red negative pressure and other symptoms of tracheal compression such as dyspnea. ${ }^{16}$ It is important to observe the coloration of the skin of the neck that may be black and blue due to swelling. It may be helpful to relieve bleeding symptoms by applying compression with cold ice packs. All observations must be documented, drainage, volume, consistency, color and functionality of the state of the drainage equipment. Control of vital signs looking for the presence of hypotension and tachycardia as these may indicate bleeding.

\section{Risk of hypocalcaemia $(5,11,16,17)$}

Hypocalcaemia is symptomatic or is not usually a very frequent complication after performing a thyroidectomy. It is usually frequent due to parathyroid injury. ${ }^{11}$

There are studies that show that calcium levels usually drop to the second or third day after surgery, so if you have been discharged it is important to do health education about the signs to be identified. ${ }^{5}$

I. Assess the presence of tremor in the extremities and numbness of the lips. In some patients, intravenous administration of $10 \%$ calcium gluconate may be necessary if the blood calcium concentration is very low. ${ }^{11}$

II. Control of plasma calcium levels in the immediate postoperative period and at discharge. ${ }^{17}$

III. Evaluation of reflexes periodically. Observe neuromuscular irritability (spasms, numbness, paraesthesia, positive signs of Chvostek and Trousseau, seizure activity). ${ }^{16}$
The Trousseau sign is manifested with carpopedal spasm by occluding the arterial blood supply of the arm for three minutes using a pressure cuff. And the Chvostek sign is manifested by the contraction of the facial muscles by striking the jaw above the facial nerve. ${ }^{17}$ There are studies that also assess parathyroid hormone in addition to calcium levels, since PTH is a reflection of parathyroid function. ${ }^{5,8}$

\section{Risk of recurrent laryngeal nerve injury $(5, \mathrm{I}, 16)$}

Laryngeal nerve injury is a serious complication of thyroid gland surgery. It can be caused by different mechanisms, including the incision, impingement, stretching of the nerve, it can also be secondary to edema or hematoma. Control the patient's voice quality, swallowing reflex and postoperative respiratory status. ${ }^{16}$ According to Xiaojuan Jian B et al. ${ }^{11}$ the appearance of hoarseness in speech and coughing when drinking is often induced by injury to the laryngeal nerve, after surgery patients were encouraged to speak and observed if there were any symptoms that included hoarseness, aphonia, dyspnea, decreased cough, cough with liquids. Patients should be guided to drink water gradually.

Furlado $\mathrm{L}^{16}$ in his review mentions that the recurrent bilateral lesion of the laryngeal nerve is the most serious complication because it results in vocal cord paralysis and with a variability in airway obstruction. Patients with this type of nerve injury, have inspiratory stridor, dyspnea, tachypnea and nasal flutter. This vocal cord paralysis can be temporary or permanent, it will be necessary to assess the performance of a tracheostomy if the vocal cords do not recover. During the postoperative period, it is important to perform an indirect laryngoscopy to assess the vocal cords and their proper functioning. ${ }^{12}$

\section{Risk of wound infection $(5,6,7,16)$}

Constant temperature control (16).

According to Balentine and Sippel (2016) ${ }^{5}$ if you have a fever in 2 readings taken 4 hours apart, increased redness and / or heat at the site of the incision, drainage in the form of pus or pain that is not controlled with painkillers there may be an infection in the neck. It is important that wounds after a thyroidectomy are observed to detect signs of infection. The infection is often caused by Staphylococcus or Streptococcus species. ${ }^{16}$

\section{Risk of infective pain management $(7,16,18)$}

The development of complications can lead to poor pain control. Therefore, it is important to recognize pain because it can be a sign of the development of some complication such as edema, infection, etc.

I. Evaluate pain, location, intensity and duration verbally and / or using scales

\section{Keep the patient in a Semi-fowler position $\left(40-35^{\circ}\right)$}

III. Keep the head and neck in a neutral position. Educate the patient to use their hands to support the neck during movement and avoid hyperextension of the neck

IV. Promote and facilitate the patient to use relaxation techniques. ${ }^{16}$

According to Ayhan $\mathrm{H}$ et al. ${ }^{19}$ after a thyroidectomy, patients experienced neck pain and disability. These problems improved spontaneously 1 month after the operation. Neck stretching exercises in the period of early post thyroidectomy facilitate neck movement and reduce pain and disability, as well as sensitivity. In addition, they 
have no direct negative effect on wound healing. Stretching exercises consist of 8 steps that should be performed 3 times a day (morning, afternoon and night)

I. Relax your shoulders and neck enough

II. Look down

III. Turn the face to the right

IV. Turn the face to the left

V. Tilt your head to the right

VI. Tilt your head to the left

VII. Turn your shoulders back and around

VIII. Raise your hands completely and then lower them.

\section{Education at discharge $(5,16,18,19)$}

Before a patient leaves, it is important that nurses identify the learning needs and knowledge deficits of patients. In this way the patient will gain security and Independence in their care. ${ }^{19}$

I. Recommend the need for a balanced diet with foods rich in calcium and vitamin D

II. Encourage an exercise program to progressively

III. Review knowledge of the medication schedule

IV. Educate about the signs and symptoms that need a medical evaluation (fever, spirits, wound drainage, erythema, significant weight loss, nausea and repeated vomiting, etc.). ${ }^{16}$

\section{Discussion and conclusions}

There are a number of known and frequent postoperative complications after a thyroidectomy such as hypocalcaemia (being the most frequent), the presence of re-bleeding leading to a hematoma with less or greater severity, recurrent laryngeal nerve paralysis. ${ }^{1,3,5,6}$, ${ }^{7,9,10}$ According to Cauley et al. ${ }^{4}$ the complications recorded at 30 days out of a total of 40,025 patients undergoing total thyroidectomy were $7.74 \%$. Preoperative factors that affected the complication rates for thyroidectomies in hospitalized patients included: age $\geq 70$ years, nonCaucasian, dependent functional status, history of congestive heart failure, history of smoking, bleeding disorder (According to Quéralt et al $17 \%$ vs. $13 \%$ of patients who performed hematoma were treated with anticoagulation) other complications were wound infection and preoperative sepsis. In addition, malignant thyroid pathology is associated with increased vascularization, immunosupression and poor wound healing. It was observed that the presence of malignancy may be a predictor of increased postoperative complications.

We must know that after performing a thyroidectomy we have to take into account that different complications may occur. The presence of high-risk cervical hematoma can lead to a compromise of the respiratory tract and death. The appearance of cervical hematoma does not have to be early can develop within days or hours after surgery and the inaccuracy of identifying patients at high risk generates anxiety for them. ${ }^{5}$ Bergenfelz et al. ${ }^{6}$ observed $2.1 \%$ of 3,660 patients who had to undergo surgery again due to re-bleeding due to compression hematoma. The risk of hematoma due to re-bleeding increased with the male sex, as did the increase in age. Not all bruises are of equal severity, some do not require reintervention and only observational control of symptoms. Another frequent complication may be the appearance of hypoparathyroidism that involves hypocalcaemia. ${ }^{5}$ Generally the management of hypocalcemia is successful with calcium supplements although some may be readmitted for starting intravenous calcium treatment in refractory cases. Calcium levels usually fall within 2 to 3 days after surgery. Bergenfelz et al. ${ }^{6}$ observed in their study that after a total thyroidectomy, hypocalcaemia occurred in $9.9 \%$ of patients and had to be treated with vitamin $\mathrm{D}$, after followup at 6 months had reduced to $4.4 \%$.

There is considerable disagreement regarding the best approach for postoperative hypoparathyroidism. There are studies that independently prescribed at discharge all patients undergoing subtotal thyroidectomy or total oral calcium therapy. In addition, patients were instructed to document and identify symptoms of paraesthesia that could be indicative of hypocalcemia. Of a total of 232 patients, $2.5 \%$ were complications related to hypocalcemia. Elderly patients had a similar rate of complications compared to young patients, including transient hypocalcemia $(12.5 \%$ vs. $11.1 \%$, respectively) and temporary vocal cord dysfunction $(2.9 \%$ vs. $3.9 \%)$, but a higher readmission rate $(4.5 \%$ vs. $1.2 \%){ }^{10}$ There are studies ${ }^{8}$ that use a more selective approach based on the measurement of calcium levels after the operation and parathyroid hormone (PTH) using a standard protocol to address the problem. Levels below $\leq 5 \mathrm{pg} / \mathrm{ml}$ may be more associated with advanced hypocalcemias with symptomatology and treatment correction.

A complication that may appear, although less frequent is the swallowing disability that may exist after a thyroidectomy, which is known as a dysfunction of the nerves that maintain laryngeal motility. It is usually secondary to the lesion of the laryngeal or inferior superior nerve. Both the upper and lower nerves give sensitivity to the posterior branches that innervate the esophagus and the cricopharyngeal muscle, which could help to understand the swallowing disability that may exist after thyroidectomy. There are different ways to diagnose this complication, by laryngoscope or endoscopy, directly observing the mobility of the vocal cords or the associated alteration of the mucosa such as Barret's esophagitis by endoscope. Also the monitoring of the $\mathrm{pH}$ changes that can occur with reflux in the esophageal mucosa. ${ }^{12}$

The degree of thyroidectomy can affect the initial results of the voice. In addition, there are some favors that can be evaluated in the early postoperative period that are the black race, a subsequent perceived atypical voice, a score of the voice disability index $>18$ points or an abnormality in the laryngeal examination can predict persistent dysphonia and therefore we can perform an early intervention to these patients. ${ }^{13}$ The vocal disability index (VHI) or Voice Handicap Index is a questionnaire in order to quantify the impact perceived by the affected subject of his voice alteration on his quality of life. ${ }^{13,20}$ Some of the limitations that should be considered in this review have to do with the search strategy, since no MeSH or DeSC terms were used, so it could have an impact on the number of documents found. On the other hand, this review has not included other documents in the scientific literature corresponding to techniques, procedures, protocols, etc. published on the subject, backed by different health institutions or scientific societies and this may be a limitation of the evidence in this study.

This review identifies the need to know the care after this intervention to ensure patient safety, as well as to prevent and detect complications. Recent technological and medical advances shorten the duration of hospitalization through more advanced surgical procedures that require a shorter hospital stay, therefore there is a tendency to ambulatory surgery and go home the same day. ${ }^{19}$. Lack of education 
at discharge, ignorance of appropriate information causes patients to experience more anxiety and feel more dependent on ignorance. Therefore, nurses must identify the learning needs and knowledge deficits of patients. This review has been carried out with the objective of knowing more about the most frequent complications after a thyroidectomy and establishing the basic learning needs that patients should know when they are discharged to take responsibility for their health. In addition, a series of recommendations have been made to patients on discharge to empower the patient to self-responsible for their health and recognize early complications, learn and take responsibility for the medication, know how to heal the wound etc. For all this, we have seen it important to guide professionals with this article to reduce the variations that may exist in clinical practice, thus promoting quality care based on evidence.

\section{Acknowledgments}

None.

\section{Conflicts of interest}

The author declares there is no conflict of interest.

\section{References}

1. Quérat C, Germain N, Dumollard J, et al. Surgical management of hyperthyroidism. Eur Ann Otorhinolaryngol Head Neck Dis. 2015;132(2):63-66.

2. Ministerio de Sanidad y Consumo y Bienestar Social. Registro de Altas de los Hospitales Generales del Sistema Nacional de Salud. CMBD. Norma Estatal. 2018.

3. Miccoli P, Frustaci G, Fosso A, et al. Surgery for recurrent goiter : complication rate and role of the thyroid-stimulating hormonesuppressive therapy after the first operation. Langenbecks Arch Surg. 2015;400(2):253-258.

4. LCaulley, S Johnson-Obsaseki, L Luo, et al. Risk factors for postoperative complications in total thyroidectomy. Medicine. 2017;96(5):6-10.

5. Balentine CJ, SippelRS. Outpatient thyroidectomy: is it safe? SurgOncol Clin N Am. 2016;25(1):61-75.

6. Bergenfelz A, Jansson S, Kristoffersson A, et al. Complicactions to thyroid surgery: results as reported in a database from a multicenter audit comprising 3.660 pacients. Langenbecks Arch Surg. 2008;393(5):667673.

7. Trottier DC, Barron P, Moonje V, et al. Outpatient thyroid surgery: should patients be discharged on the day of their procedures? Can $J$ Surg. 2009;52(3):182-186.

8. Carter Y, Chen H, Sippel RS. An intact parathyroid hormone-based protocol for the prevention and treatment of symptomatic hypocalcemia after thyroidectomy. J Surg Res. 2014;186(1):23-28.

9. Melanie WS, David JT. Geriatric Thyroidectomy: Safety of Thyroid Surgery in Aging Population. JAMA Arch Otolaryngol Head Neck Surg. 2009; 135(10):1041-44.

10. Efremidou EM, Papageorgiou MS. The efficacy and safety of total thyroidectomy in the management of benign thyroid disease : a review of 932 cases. Can J Surg. 2009;52(1):39-44.

11. Xiaojuan Jian, Bo Gao, Jiaqun Zou, et al. Perioperative Nursing and Intervention of Postoperative Complications for Thyroidectomy. Acta Medica Med. 2014;30(1):355-360.

12. Scerrino G, Tudisca C, Bonventre S, et al. Swallowing disorders after thyroidectomy: What we know and where we are: a systematic review. Int J Surg. 2017;41:S94-S102.

13. Vicente DA, Solomon NP, Avital I, et al. Voice Outcomes after Total Thyroidectomy, Partial Thyroidectomy, or Non-Neck Surgery Using a Prospective Multifactorial Assessment. J Am Coll Surg. 2014;219(1):152-63.

14. CASPe (Critical Appraisal Skills Programme Español). Alicante (España). Programa de Habilidades en Lectura Crítica Español. 2007.

15. Jordan Z, Lockwood C, Aromataris E, et al. The updated JBI model for evidence-based healthcare. Int J Evid Based Healthc. 2019;17(1):58-71.

16. Furtado L. Thryroidectomy: post-operative care and common complications. Nursing Standard. 2011;25(34):43-52.

17. Montañez JB, Carrasco CR. Postoperatorio inmediato en pacientes sometidos a tiroidectomía total en UCI Immediate post-operative period in patients undergoing total thyroidectomy in the ICU. Enfermería Intensiva. 2002;13(2):78-84

18. Ayhan H, Tastan S, Iyigun E, et al. The effectiveness of the neck exercises following total thyroidectomy on reducing neck pain and disability: Randomized controlled trial. Cancer Nurs. 2015;38(4):224-231.

19. Temiz Z, Ozturk D, Ugras GA, et al. Determination of patient learning needs after thyroidectomy. Asian Pacific J Cancer Prev. 2016;17(3):1479-1483.

20. Solomon NP, Helou LB, Henry LR, et al. Utility of the Voice Handicap Index as an Indicator of Postthyroidectomy Voice Dysfunction. $J$ Voice. 2013;27(3):348-354. 